\title{
A retrospective analysis of radiotherapy in the treatment of external auditory canal carcinoma
}

\author{
ATSUTO KATANO $^{1}$, RYOUSUKE TAKENAKA ${ }^{1}$, HIDEOMI YAMASHITA ${ }^{1}$, MIZUO ANDO ${ }^{2}$, \\ MASAFUMI YOSHIDA ${ }^{2}$, YUKI SAITO ${ }^{2}$, TAKAHIRO ASAKAGE ${ }^{3}$, OSAMU ABE $^{1}$ and KEIICHI NAKAGAWA ${ }^{1}$ \\ Departments of ${ }^{1}$ Radiology or ${ }^{2}$ Otolaryngology, The University of Tokyo Hospital, Bunkyo-ku, Tokyo 113-8655; \\ ${ }^{3}$ Department of Head and Neck Surgery, Tokyo Medical and Dental University, Tokyo 113-8510, Japan
}

Received September 24, 2020; Accepted November 13, 2020

DOI: $10.3892 / \mathrm{mco} .2021 .2207$

\begin{abstract}
External auditory canal carcinoma (EACC) is a rare disease. The current study aimed to evaluate the clinical outcomes of patients treated with external beam radiotherapy (EBRT) for EACC. The present study retrospectively reviewed 34 consecutive patients treated for EACC with EBRT between February 2001 and January 2019 at the University of Tokyo Hospital. Clinical staging was performed according to the modified Pittsburgh classification. Of all the included patients, seven patients were in the early stages (I or II) and 27 in the advanced stages (III or IV) of EACC. A total of 16 patients underwent EBRT and surgery (S+RT) pre- and/or postoperatively, while 18 patients underwent definitive radiotherapy (dRT). The median prescribed doses for the S+RT and dRT groups were 66 and $70 \mathrm{~Gy}$, respectively. The median follow-up period for all patients was 22.4 months (range, 2-205 months). The 5 -year overall survival rates of the S+RT and dRT groups were 66.7 and $45.1 \%$, respectively. The progression-free survival rate at 5-year was $55.6 \%$ (95\% confidence interval: $36.5-71.1 \%$ ) for the entire cohort. A total of 14 patients experienced disease relapse after treatment, consisting of 11 locoregional recurrences and three distant metastases. The current study revealed the clinical outcomes of EBRT for EACC.
\end{abstract}

\section{Introduction}

External auditory canal carcinoma (EACC) is an extremely rare disease with an incidence of approximately one in a million and accounts for $<1 \%$ of all head and neck cancers $(1,2)$. EACC often requires substantial clinical time from the onset of symptoms to the diagnosis because of its low incidence. The initial clinical symptoms of EACC are nonspecific and include otorrhea, tinnitus, otalgia, hearing loss, clogged ears,

Correspondence to: Dr Hideomi Yamashita, Department of Radiology, The University of Tokyo Hospital, 7-3-1 Hongo, Bunkyo-ku, Tokyo 113-8655, Japan

E-mail: yamachan07291973@yahoo.co.jp

Key words: external auditory canal carcinoma, ear, radiotherapy, treatment outcome, retrospective analysis and bleeding. In patients with advanced stages of EACC, facial paralysis is also present and is associated with poor survival. Chronic stimulation, such as habitual ear picking, has also been recognized as an important factor in the carcinogenesis of EACC (3).

Squamous cell carcinoma is the most common histological type of EACC and accounts for $80-90 \%$ of cases, followed by adenoid cystic carcinoma, basal cell carcinoma, and adenocarcinoma (4). Several criteria have been formulated in the clinical staging system of EACC. According to the 8th edition of the American Joint Committee on Cancer staging manual on the tumor, node, and metastasis staging system, EACC is classified as cutaneous cancers of the head and neck. The Stell and McCormick staging system is based on staging of external auditory canal and middle ear carcinomas (5). The modified Pittsburgh staging system (6) is also widely used in external auditory squamous cell carcinoma, which originates from the Arriaga staging system (7) based on preoperative clinical examination and computed tomography (CT) findings. Although there is no universally accepted staging system, the modified Pittsburgh staging system is valuable for clinical decision-making (8).

Surgery is the mainstay of treatment for early stage EACC (9). Radiotherapy is indicated in the following cases: i) Radical treatment for patients unable to undergo surgery, ii) postoperative treatment for patients who have undergone surgical resection, but having a high risk of recurrence (e.g., positive margins and locally advanced cases), and iii) preoperative treatment for medically operable and locally advanced cases. Multimodal treatment combining local and systemic treatment approaches is advised for locally advanced cases; however, optimal treatment remains controversial.

Performing a large statistical examination is often difficult in a single institution because of the limited number of cases. Hence, careful consideration of these cases is important. This retrospective study aimed to evaluate the feasibility and efficacy of external beam radiotherapy (EBRT) with or without surgery for patients with EACC. We reviewed approximately 20 years of EACC treatment in our institution.

\section{Materials and methods}

We retrospectively reviewed 34 consecutive patients with EACC treated by curative EBRT with or without surgery between 
February 2001 and January 2019. The inclusion criteria were as follows: histologically confirmed cases of primary EACC, cases treated using EBRT with curative intent, cases with no evidence of distant metastasis at the time of diagnosis, and no prior radiotherapy to the temporal bone region. The exclusion criteria were as follows: Radiotherapy for palliative intent cases and lack of sufficient medical records to confirm EACC or to decide the treatment modality. The clinical staging was performed according to the modified Pittsburgh staging system, because a recent study had been reported the clinical usefulness of that staging system (8). Acute and late adverse events were graded according to the National Cancer Institute Common Toxicity Criteria for Adverse Events, version 4.0.

$\mathrm{CT}$ of the patients for planning the radiation therapy was performed while wearing a thermoplastic mask. All patients underwent radiotherapy with 4-6 MV photon linear accelerators by radiation technologies of three-dimensional conformal radiotherapy (3D-CRT), static intensity-modulated radiotherapy (IMRT), or volumetric modulated arc therapy (VMAT). The CT image data were reconstructed with a slice thickness of $5 \mathrm{~mm}$ for 3D-CRT and $2 \mathrm{~mm}$ for IMRT or VMAT. The clinical target volume (CTV) included the primary tumor and clinically positive lymph nodes. The CTV included the tumor bed for the postoperative cases. The planning target volume included the CTV with a minimum added margin of 3-5 $\mathrm{mm}$. No patient received prophylactic irradiation for the clinically negative neck.

Statistical analysis. The $\mathrm{R}$ statistical package (The $\mathrm{R}$ Foundation for Statistical Computing, Vienna, Austria) was used for data analyses. For comparison of the proportions of patient's characteristics in two groups, the Fisher exact test was used. Overall survival (OS), progression-free survival (PFS), and cancer-specific survival (CSS) rates were calculated from the first day of initial therapy by the Kaplan-Meier method. PFS was defined as the time from initial therapy to first evidence of radiological or clinical tumor progression, or until death from any cause. A univariate Cox proportional hazard analysis was performed, since a small sample size made it difficult to conduct a multivariate analysis to reach a valid conclusion. The P-values of $<0.05$ were considered statistically significant.

\section{Results}

In this study, 34 patients were retrospectively analyzed. The median follow-up period for these patients was 22.4 months (range: 2-205 months). The pathological diagnosis of all patients was predominantly squamous cell carcinoma (31 patients), followed by adenoid cystic carcinoma (1 patient), undifferentiated carcinoma (1 patient), and poorly differentiated adenocarcinoma (1 patient). Of all the patients, 18 received definitive radiotherapy (dRT) and 16 received EBRT combined with surgery $(\mathrm{S}+\mathrm{RT})$. The median follow-up period for the dRT and S+RT groups was 15.3 (range: 2-205 months) and 75.1 months (range: 5-169 months), respectively. Patient characteristics for both the groups are presented in Table I. The S+RT group had a higher ratio of male patients and better Karnofsky Performance Status (KPS) compared to the dRT group. No statistically significant difference was observed between the two groups in terms of patient age, clinical stage, pathology, and prescribed dose. The entire cohort consisted of 19 men and 15 women, with a median age of 66 years (range: 32-86 years). According to the modified Pittsburgh staging system, $21 \%$ of the patients were classified in early stage and $79 \%$ in advanced stage of EACC.

The radiotherapy characteristics of the patients are shown in Table II. In the dRT group, 13 patients underwent EBRT concurrently with chemotherapy or biotherapy, which consisted of the docetaxel platinum plus 5-fluorouracil (DCF) regimen, platinum plus 5-fluorouracil (CF) regimen, single-agent platinum regimen, and cetuximab.

The S+RT group underwent postoperative radiotherapy $(n=10)$ and pre- and/or postoperative radiotherapy $(n=6)$. According to the surgical treatment modalities, one patient underwent mastoidectomy, seven patients underwent lateral temporal bone resection, and eight patients underwent subtotal temporal bone resection. All patients who received postoperative radiotherapy underwent EBRT without chemotherapy. In total, there were six patients in the pre- and/or postoperative radiotherapy group; four patients received radiation therapy alone (without chemotherapy), one patient received a daily low-dose of cisplatin concurrently undergoing the pre- and/or postoperative radiation therapy, and one patient received neoadjuvant chemotherapy consisting of cisplatin and 5-fluorouracil prior to preoperative radiotherapy. The median prescribed doses for the S+RT and dRT groups were 66 (range: 50-70 Gy) and $70 \mathrm{~Gy}$ (range: 60-70 Gy), respectively.

Of all the patients, $19(56 \%)$ patients were alive at the time of this analysis; the 5-year OS and CSS rates for the entire cohort were 55.2 (95\% confidence interval [CI]: 35.7-71.1\%) and $57.1 \%$ (95\% CI: 37.0-72.9\%), respectively (Fig. 1). The 5 -year OS rates for the early and advanced stages were 85.7 and $45.6 \%$, respectively (Fig. 2A). There was no significant statistical difference between the 5-year OS rates of both the groups (Fig. 2B, 66.7 vs. 45.1\%, P=0.104). With reference to the univariate analysis (Table III), a factor associated with a favorable OS rate included a good performance status of over or equal to $90 \%$ (hazard ratio: $0.137,95 \%$ CI: $0.043-0.434$, and $\mathrm{P}<0.001)$.

Of all the patients, $14(41.2 \%)$ developed recurrences, including nine with local recurrences, two with combined locoregional recurrences, and three with distant metastases. The PFS rate at 5-year was 55.6\% (95\% CI: 36.5-71.1\%) for the entire cohort. A wide variety of metastatic sites, including the lungs, liver, and bone, was observed in cases with distant metastases. Salvage surgery was performed in one patient, while chemotherapy was performed in three patients for patients with recurrence. The remaining patients selected the option of best supportive care. Among the patients treated with chemotherapy, one patient received the $\mathrm{CF}$ regimen and two patients received oral $\mathrm{S}-1$ administration. The median survival time after recurrence in patients with recurrence was 2.9 months (range: 0.2-23 months). Severe (Grade 3 or higher) radiation-induced late complications were not observed.

\section{Discussion}

Our study evaluated the clinical outcomes of patients treated with EBRT for EACC. Our results were consistent with those of previous reports. A meta-analysis of 742 patients reported 
Table I. Patient characteristics of 34 patient with external auditory canal carcinoma in our institution.

\begin{tabular}{|c|c|c|c|}
\hline Characteristics & dRT group $(n=18)$ & S+RT group $(n=16)$ & P-value \\
\hline Age, median (range) & $68(46-86)$ & $65(32-75)$ & 0.925 \\
\hline Sex & & & 0.045 \\
\hline Male & $7(39 \%)$ & $12(75 \%)$ & \\
\hline Female & $11(61 \%)$ & $4(25 \%)$ & \\
\hline KPS, median (range) & $80(70-100)$ & $90(80-100)$ & 0.008 \\
\hline Clinical stage & & & 0.214 \\
\hline Early (I/II) & $2(11 \%)$ & $5(31 \%)$ & \\
\hline Advanced (III/IV) & $16(89 \%)$ & $11(69 \%)$ & \\
\hline Pathology & & & 0.591 \\
\hline $\mathrm{SqCC}$ & $17(94 \%)$ & $14(88 \%)$ & \\
\hline $\mathrm{ACC}$ & 0 & $1(6 \%)$ & \\
\hline Others & $1(6 \%)$ & $1(6 \%)$ & 0.446 \\
\hline Prescribed dose, median (range) & 70 Gy (50-70 Gy) & 66 Gy $(60-70 \mathrm{~Gy}]$ & \\
\hline
\end{tabular}

KPS, Karnofsky Performance Status; dRT, Definitive radiotherapy; S+RT, surgery plus radiotherapy; SqCC, Squamous cell carcinoma; ACC, Adenoid cystic carcinoma; Gy, gray (a derived unit of ionizing radiation dose).

Table II. Radiotherapy characteristics of 34 patient with external auditory canal carcinoma in the University of Tokyo Hospital.

\begin{tabular}{lrc}
\hline Treatment type & N & Percentage (\%) \\
\hline Definitive radiotherapy & 18 & 53 \\
Bioradiotherapy (cetuximab) & 1 & \\
CCRT & 12 & \\
Docetaxel + Cisplatin + 5FU (DCF) & 8 & \\
Cisplatin + 5-FU (CF) & 2 & \\
Daily Cisplatin & 2 & \\
RT alone & 5 & \\
Radiotherapy with surgery & 16 & 47 \\
Postoperative (RT alone) & 10 & \\
Pre + postoperative & 6 & \\
RT alone & 4 \\
Chemoradiotherapy (including NAC) & 2
\end{tabular}

CCRT, concurrent chemoradiotherapy; NAC, neoadjuvant chemotherapy; 5-FU, 5-fluorouracil; DCF, docetaxel cisplatin plus 5-fluorouracil; CF, cisplatin plus 5-fluorouracil; RT, radiotherapy.

that patients with external auditory canal squamous cell carcinoma treated with chemoradiotherapy had a similar survival rate (10). A multi-institutional review of 87 patients that focused primarily on the roles of surgery and radiotherapy in these patients discovered that the 5-year OS and disease-free survival rates were 55 and 54\%, respectively (11). Furthermore, this review indicated that the clinical stage and treatment modality were significant prognostic factors.

To the best of our knowledge, there are no prospective or randomized trials about EACC treatment. For the patient in early stage EACC, surgical resection with tumor free margin is the most standard treatment $(12,13)$. Radical conventional radiotherapy also achieves favorable outcome, which was considered as treatment option (14). Murai et al reported the effectiveness of the stereotactic radiotherapy for a new treatment option of EACC (15).

However, there is no clear consensus about treatment strategies in advanced cases. Surgical resection in combination with perioperative radiotherapy is more effective compared to a single modality treatment (16-18). Nakagawa et al used preoperative radiotherapy; their study revealed that the tumor-free surgical margin had a significant correlation with patient survival in locally advanced cases (19). In another study conducted by Choi et al they considered the necessity of postoperative radiotherapy in accordance with the clinical stage (20). The disease control rates for patients with early vs. advanced stages were 55.6 vs. $50 \%$ in the postoperative radiotherapy group and 66.7 vs. $37.5 \%$ in the dRT group. In this study, while the 5-year OS rate was higher in the S+RT group, the difference did not reach statistical significance (66.7 vs. $45.1 \%, \mathrm{P}=0.104)$.

Despite the aggressive multidisciplinary treatment, locoregional failure remains the most frequent recurrence; Yoon et al reported a high propensity for locoregional failure of EACC (21). Furthermore, they reported nine local recurrences, eight regional recurrences, and three distant metastases among 20 patients with recurrence. In our study, locoregional failure was detected in $76 \%$ of patients with recurrence. Therefore, a more aggressive local therapy could be beneficial to some extent. Hayashi et al reported the clinical outcome of carbon ion radiotherapy for external auditory canal and middle ear carcinomas in a retrospective multicenter study (22). They revealed that the 3 -year local control and OS rates were 55 and $59 \%$, respectively.

The optimal chemotherapy regimen for concurrent chemoradiotherapy in definitive radiotherapy is still 
Table III. Univariate Cox proportional hazard analysis of overall survival.

\begin{tabular}{|c|c|c|c|c|}
\hline Covariables & $\mathrm{N}$ & Hazard ratio & $95 \% \mathrm{CI}$ & P-value \\
\hline \multicolumn{5}{|l|}{ Age } \\
\hline$\leq 65$ years old & 16 & 1 & & \\
\hline$>65$ years old & 18 & 1.529 & $0.542-4.312$ & 0.422 \\
\hline \multicolumn{5}{|l|}{ Sex } \\
\hline Female & 15 & 1 & & \\
\hline Male & 19 & 0.647 & $0.234-1.788$ & 0.401 \\
\hline \multicolumn{5}{|c|}{ Performance status } \\
\hline$<90 \%$ & 10 & 1 & & \\
\hline$\geq 90 \%$ & 24 & 0.137 & $0.043-0.434$ & $<0.001$ \\
\hline \multicolumn{5}{|l|}{ Clinical stage } \\
\hline Early & 7 & 1 & & \\
\hline Advanced & 27 & 2.493 & $0.558-11.130$ & 0.231 \\
\hline \multicolumn{5}{|c|}{ Pathological type } \\
\hline $\mathrm{SqCC}$ & 31 & 1 & & \\
\hline non $\mathrm{SqCC}$ & 3 & 0.976 & $0.127-7.525$ & 0.982 \\
\hline \multicolumn{5}{|l|}{ Surgery } \\
\hline No & 18 & 1 & & \\
\hline Yes & 16 & 0.406 & $0.137-1.204$ & 0.104 \\
\hline \multicolumn{5}{|l|}{ Chemotherapy } \\
\hline No & 20 & 1 & & \\
\hline Yes & 14 & 1.300 & $0.460-3.679$ & 0.621 \\
\hline
\end{tabular}

CI, confidence interval; SqCC, Squamous cell carcinoma.
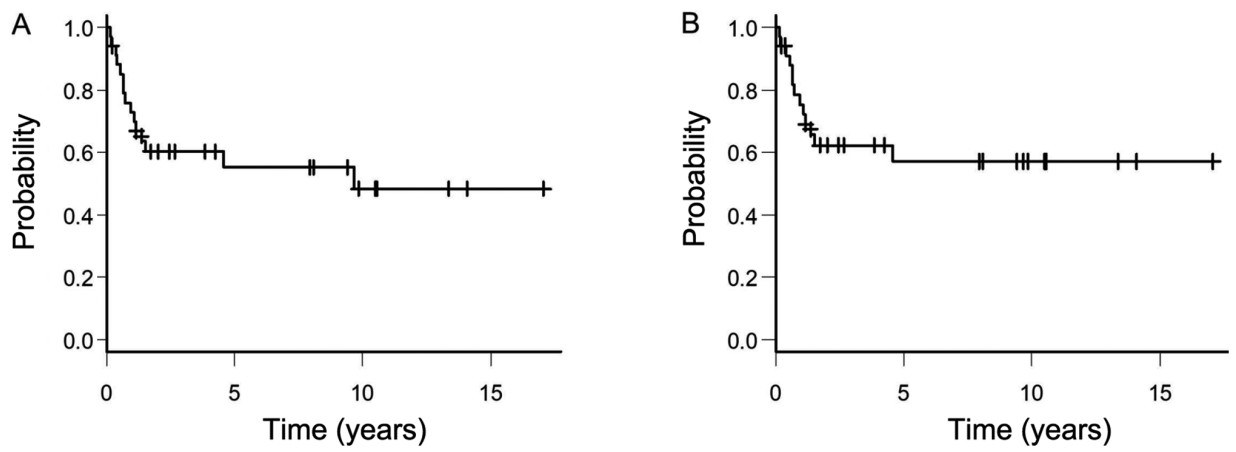

Figure 1. Kaplan-Meier curve for (A) overall survival and (B) cancer-specific survival in patients with external auditory canal carcinoma treated in our institution. A vertical bar indicates a censored case.
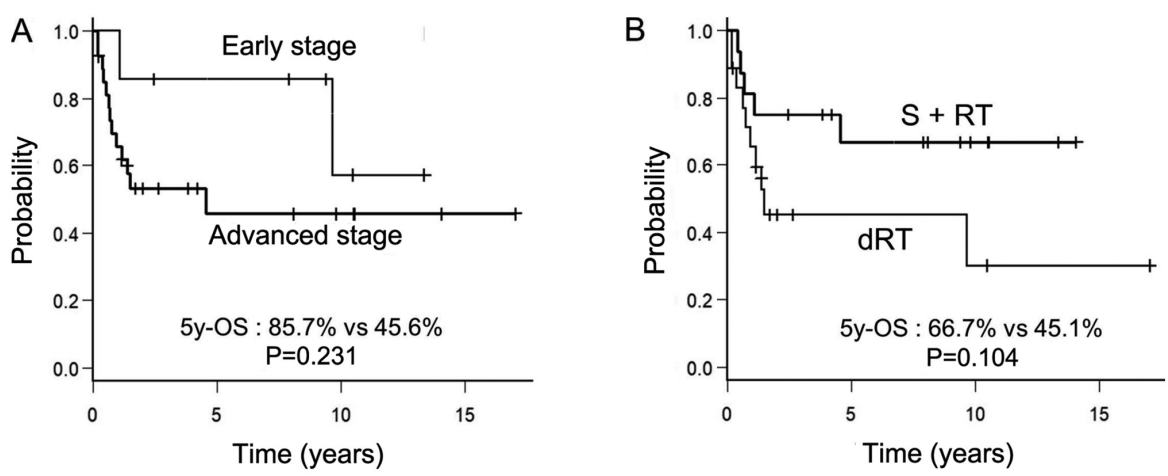

Figure 2. Kaplan-Meier curves for (A) overall survival stratified clinical stage: Early vs. advanced) and (B) treatment strategy: dRT vs. S+RT. A vertical bar indicates a censored case. dRT, Definitive radiotherapy; S+RT, surgery plus radiotherapy; OS, overall survival. 
controversial. Several anticancer drugs, such as cisplatin, carboplatin, fluorouracil, docetaxel, and mitomycin, are widely used (10). Nagano et al reported that the DCF regimen was potentially the most effective method, with reference to their study that revealed a $100 \%$ 2-year OS and locoregional control rate in six patients with advanced EACC who had received this regimen (23). Other studies supported the use of an intra-arterial cisplatin infusion in combination with radiotherapy for locally advanced cases of EACC $(24,25)$.

Several limitations existed in our study. First, the most important limitation of present study is the low number of cases and the retrospective nature of the study could have produced a selection bias. Second, a long observation period resulted in a substantial heterogeneity of treatment modality, such as chemotherapy regimens, treatment modality, radiation technology, and surgical procedure. Third, there was unclearness about decision making process of the therapeutic strategies at the time of the treatment because of retrospective data analysis. Fourth, our study included different pathological types, which could have caused inaccurate results in the study. Fifth, our data were dependent on the medical records, which may be insufficient for accurately describing all the patient characteristics and events, especially in adverse event detection. This factor limited the availability of patient covariates, and unmeasured confounding variables most likely existed during the study.

In conclusion, our retrospective study reported the clinical outcomes of EACC in our institution. No statistically significant difference between the dRT and $\mathrm{S}+\mathrm{RT}$ groups was observed. However, the S+RT group had a better prognosis tendency than the dRT group, which was consistent with a previous report. Further studies and accumulation of data are needed to determine the optimal treatment strategy for EACC in the future.

\section{Acknowledgements}

Not applicable.

\section{Funding}

No funding was received.

\section{Availability of data and materials}

All data generated or analyzed during this study are included in this published article.

\section{Authors' contributions}

AK was a major contributor to writing this report. AK collected and assembled the data. HY and AK analyzed and interpreted the clinical data. HY conceived of this study and participated in its design and coordination. RT, MA, MY, TA and YS contributed to the acquisition of data. OA and KN made substantial contributions to interpretation of data. RT, HY, MA, MY, YS, TA, OA and KN revised this report critically for important intellectual content. All authors have read and approved the final manuscript.

\section{Ethics approval and consent to participate}

Written informed consent was obtained from all patients. The current study was approved the institutional review board in the University of Tokyo Hospital (Tokyo, Japan).

\section{Patient consent for publication}

Not applicable.

\section{Competing interests}

The authors declare that they have no competing interests.

\section{References}

1. Kuhel WI, Hume CR and Selesnick SH: Cancer of the external auditory canal and temporal bone. Otolaryngol Clin North Am 29: 827-852, 1996.

2. Nyrop M and Grontved A: Cancer of the external auditory canal. Arch Otolaryngol Head Neck Surg 128: 834-837, 2002.

3. Tsunoda A, Sumi T, Terasaki O and Kishimoto S: Right dominance in the incidence of external auditory canal squamous cell carcinoma in the Japanese population: Does handedness affect carcinogenesis? Laryngoscope Investig Otolaryngol 2: 19-22, 2017.

4. Devaney KO, Boschman CR, Willard SC, Ferlito A and Rinaldo A: Tumours of the external ear and temporal bone. Lancet Oncol 6: 411-420, 2005.

5. Stell PM and McCormick MS: Carcinoma of the external auditory meatus and middle ear. Prognostic factors and a suggested staging system. J Laryngol Otol 99: 847-850, 1985.

6. Moody SA, Hirsch BE and Myers EN: Squamous cell carcinoma of the external auditory canal: An evaluation of a staging system. Am J Otol 21: 582-588, 2000.

7. Arriaga M, Curtin H, Takahashi H, Hirsch BE and Kamerer DB: Staging proposal for external auditory meatus carcinoma based on preoperative clinical examination and computed tomography findings. Ann Otol Rhinol Laryngol 99: 714-721, 1990.

8. Morita S, Mizumachi T, Nakamaru Y, Sakashita T, Kano S, Hoshino K, Fukuda A, Fujiwara K and Homma A: Comparison of the university of pittsburgh staging system and the eighth edition of the american joint committee on Cancer TNM classification for the prognostic evaluation of external auditory canal cancer. Int J Clin Oncol 23: 1029-1037, 2018.

9. Matoba T, Hanai N, Suzuki H, Nishikawa D, Tachibana E, Okada T, Murakami S and Hasegawa Y: Treatment and outcomes of carcinoma of the external and middle Ear: The validity of En bloc resection for advanced tumor. Neurol Med Chir (Tokyo) 58: 32-38, 2018.

10. Takenaka Y, Cho H, Nakahara S, Yamamoto Y, Yasui T and Inohara $\mathrm{H}$ : Chemoradiation therapy for squamous cell carcinoma of the external auditory canal: A meta-analysis. Head Neck 37: 1073-1080, 2015.

11. Ogawa K, Nakamura K, Hatano K, Uno T, Fuwa N, Itami J, Kojya S, Nakashima T, Shinhama A, Nakagawa T, et al: Treatment and prognosis of squamous cell carcinoma of the external auditory canal and middle ear: A multi-institutional retrospective review of 87 patients. Int J Radiat Oncol Biol Phys 68: 1326-1334, 2007.

12. Bacciu A, Clemente IA, Piccirillo E, Ferrari S and Sanna M: Guidelines for treating temporal bone carcinoma based on long-term outcomes. Otol Neurotol 34: 898-907, 2013.

13. Fleiner F, Jumah M and Göktas O: Cancer of the external auditory canal-diagnostic and treatment. Indian J Otolaryngol Head Neck Surg 61: 270-274, 2009.

14. Pemberton LS, Swindell R and Sykes AJ: Primary radical radiotherapy for squamous cell carcinoma of the middle Ear and external auditory Cana-an historical series. Clin Oncol (R Coll Radiol) 18: 390-394, 2006.

15. Murai T, Kamata SE, Sato K, Miura K, Inoue M, Yokota N, Ohta S, Iwabuchi M, Iwata H and Shibamoto Y: Hypofractionated stereotactic radiotherapy for auditory canal or middle ear cancer. Cancer Control 23: 311-316, 2016. 
16. Shinomiya H, Hasegawa S, Yamashita D, Ejima Y, Kenji Y, Otsuki N, Kiyota N, Sakakibara S, Nomura T, Hashikawa K, et al: Concomitant chemoradiotherapy for advanced squamous cell carcinoma of the temporal bone. Head Neck 38 (Suppl 1): E949-E953, 2016.

17. Austin JR, Stewart KL and Fawzi N: Squamous cell carcinoma of the external auditory canal. Therapeutic prognosis based on a proposed staging system. Arch Otolaryngol Head Neck Surg 120: 1228-1232, 1994

18. Hahn SS, Kim JA, Goodchild N and Constable WC: Carcinoma of the middle ear and external auditory canal. Int J Radiat Oncol Biol Phys 9: 1003-1007, 1983.

19. Nakagawa T, Kumamoto Y, Natori Y, Shiratsuchi H, Toh S, Kakazu Y, Shibata S, Nakashima T and Komune S: Squamous cell carcinoma of the external auditory canal and middle Ear: An operation combined with preoperative chemoradiotherapy and a free surgical margin. Otol Neurotol 27: 242-249, 2006.

20. Choi J, Kim SH, Koh YW, Choi EC, Lee CG and Keum KC: Tumor stage-related role of radiotherapy in patients with an external auditory canal and middle Ear carcinoma. Cancer Res Treat 49: 178-184, 2017.

21. Yoon M, Chougule P, Dufresne R and Wanebo HJ: Localized carcinoma of the external ear is an unrecognized aggressive disease with a high propensity for local regional recurrence. Am J Surg 164: 574-577, 1992.
22. Hayashi K, Koto M, Demizu Y, Saitoh JI, Suefuji H, Okimoto T, Ohno T, Shioyama Y, Takagi R, Ikawa $\mathrm{H}$, et al: A retrospective multicenter study of carbon-ion radiotherapy for external auditory canal and middle Ear carcinomas. Cancer Med 8: 51-57, 2019.

23. Nagano T, Yoshimura RI, Kojima M, Nakagawa K and Toda K: Outcomes of radiotherapy in advanced external auditory canal cancer. J Radiat Res 60: 380-386, 2019.

24. Sugimoto H, Ito M, Yoshida S, Hatano M and Yoshizaki T: Concurrent superselective intra-arterial chemotherapy and radiotherapy for late-stage squamous cell carcinoma of the temporal bone. Ann Otol Rhinol Laryngol 120: 372-376, 2011.

25. Fujiwara M, Yamamoto S, Doi H, Takada Y, Odawara S, Niwa Y, Ishikura R, Kamikonya N, Terada T, Uwa N, et al: Arterial chemoradiotherapy for carcinomas of the external auditory canal and middle ear. Laryngoscope 125: 685-689, 2015. 